\title{
Comments on the behaviour of a fixed-bed reactor
}

\author{
(Received 30 August 1990; accepted 23 April 1991)
}

\section{Dear Sirs,}

In a recent series of papers by Morbidelli and Varma and co-workers (see e.g. Morbidelli and Varma 1986a; Morbidelli et al., 1986b; Lee and Varma, 1987, 1988; Bauman and Varma 1990), the multiplicity behaviour of a fixed bed reactor is discussed. The basis for this analysis was given by Morbidelli and Varma (1986a) and Morbidelli et al. (1986b), where the authors devote many columns to mathematically prove, by means of "a rigorous application of the Implicit Function Theorem", that the multiplicity of a heterogeneous plug flow packed bed reactor is fully determined by the multiplicity of the inlet particle. Thus, according to Morbidelli et al, this model even in its basic form, does not exhibit an infinite number of steady states, a property re. ferred to as infinite multiplicity (Liu and Amundson, 1962; Eigenberger, 1972).

Eigenberger (1972) showed quantitatively that infinite multiplicity will not occur if axial dispersion of heat is present. Morbidelli et al claim that they have proven that, in order to exclude infinite multiplicity, it is not necessary to take into account axial dispersion. We will show that their proof is incorrect and that the modelling equations of the heterogeneous plug flow reactor without thermal axial dispersion do have an infinite number of steady-state solutions. The notation and the equations can be found in Morbidelli and Varma (1986a).

The solution of the heterogeneous plug flow model involves the integration of an ordinary differential equation, eq. (7), where at each step in the integration, the non-linear constraint eq. (17)-representing the state of the particlesneeds to be solved to yield $\theta_{p}$. As is well known, there can be either one or two stable solutions for this constraint. If the particles operate in the multiplicity region, then there are two possible solutions for the constraint eq. (17). In each step of the integration we must choose between these two solutions. In other words, we must choose the particle to be either in the lower or in the higher state and during the integration we must make this choice for each individual particle over and over again. Now, according to Morbidelli et al. we cannot choose arbitrarily between these two states. They concluded this from the following statement: "from the implicit function theorem it can be seen that the obtained solution of eq. (17) is unique and continuous as long as the following condition is satisfied:

$$
\frac{\mathrm{d} f}{\mathrm{~d} \overline{\theta_{p}}} \neq 0
$$

only when the condition in eq. (18) is violated, is a discontinuity in the $\theta_{p}$ solution of eq. (17) admissible". This means that once we have chosen between the upper and lower solution at the start of the integration, we must choose that same solution for each following step in the integration, until condition of eq. (18) is violated. From this it immediately follows that the multiplicity of the reactor is fully determined by the multiplicity of the particle at the reactor inlet.

However, this is not correct. From the implicit function theorem it follows that, if $\mathrm{d} f\left(\theta_{m}, \theta, z\right) / \mathrm{d} \theta_{n} \neq 0$ for some point $\left(\theta_{p}^{\circ}, \theta^{\circ}, z^{\circ}\right)$ then there exists an interval $\theta_{p}^{1}<\theta_{p}<\theta_{p}^{2}$ containing $\theta_{p}^{n}$ such that $\theta_{p}$ is uniquely defined as a function of $\theta$ and $z$. Note that this theorem only states that there exists such an interval, but does not specify $\theta_{p}^{1}$ and $\theta_{p}^{2}$. It follows that if $\mathrm{d} f\left(\theta_{p}, \theta, z\right) / \mathrm{d} \theta_{p} \neq 0$ for $\left(\theta_{p}, \theta^{\circ}, z^{\circ}\right)$ for all feasible values of $\theta_{p}$, then $\theta_{p}$ is uniquely defined by $\theta$ and $z$ in some region containing $\theta^{\circ}$ and $z^{0}$ and from this we see that the implicit function theorem provides a sufficient - but not necessarycondition for uniqueness, in other words whether or not a particle has only one steady state. Additionally, if we always choose the solution from the same branch, then with the aid of the implicit function theorem we can indeed prove that the obtained $\theta_{x}$-profile is continuous as long as the condition of eq. (18) is not violated. And also, in that case, with the aid of the existence and uniqueness theorem for initial value differential equations (Morbidelli et al., 1986b) we can prove that there exists a unique solution for the differential equation in an interval around the inlet. However, it is important to note that, if there is more than one solution of eq. (17), the implicit function theorem does not help us in making a choice between them: any one solution is permissible and so we do not have to choose the solutions to lie on the same branch! Thus, during the integration we can chouse arbitrarily between the two solutions of eq. (17), implying that a jump is allowed at any place and, therefore, the model equations do have an infinite number of solutions for some range of parameter values.

In a physical sense, the line of reasoning of Morbidelli et $a$ i. is erroneous because they had confused the steady-state behaviour of the catalyst particle with its dynamic behaviour. From a steady-state analysis discussed herc, we can deduce how many stable steady states there are, but of course we cannot determine in which of the possible states the particles are actually operating, because this depends on the particles' history, which is not included in the steadystate model. There is no mathematical law that dictates us which of the two solutions of eq. (17) we must choose. Correspondingly, in the hypothetical physical system defined by the model equations, there is no law that forbids us to choose arbitrarily between the upper and lower steady state. Thus, a temperature profile with a discontinuous jump at any place, or even a profile with more than one jump can be the steady state of the hypothetical system. It cannot be the steady state of a real fixed reactor because, contrary to the hypothetical system, in a real reactor the state of one pellet has an influence on its neighboring pellets due to heat transfer effects. If one pellet is in the ignited state, then also its neighboring pellets will ignite if they operate in the multiple steady-state region.

In order to get rid of infinite multiplicity, we must alter our model. This can be done by incorporating axial conduction. It can also be done by defining additional constraints. In a way this is what Morbidelli et al. did. If we take the original plug flow model equations, but in addition to this we formulate Morbidelli's condition quoted above, then we have got rid of infinite multiplicity. A possible physical justification for this addition to the model could be: the relevant steady state of a reactor is the state that is also attained in a dynamic situation after large times. If we assume that at an initial time all pellets operate in the lower operating point, then in the steady state that will cventually be reached, only the pellets for which the ignition temperature is exceeded, will be in the ignited state. If this ignition temperature is exceeded for the first pellet, then all pellets will be ignited. A lowering of the inlet temperature, below the extinction temperature, will result in a situation where only the pellets for which the extinction temperature is still exceeded will operate in the higher steady state. This gives rise again to an infinite number of steady states, and so we see that this new model, 
and thus Morbidelli's in general, describes only the case where the steady state is approached from the lower side, because in their model a jump is only allowed at the ignition temperature.

In the above paragraph we have tried to provide a new basis for the work done by Morbidelli, Lee and Varma in their series of papers, where they needed to exclude infinite multiplicity because that would impede a complete analysis. Contrary to their claim, the models studied by them are not the models in their basic form, but these contain an additional restriction, which in general causes all pellets to be in the lower operating point, except those for which the ignition temperature is exceeded: a jump inside the reactor is only allowed at the ignition temperature. However, we feel that this assumption is not very solid if the ignition temperature is actually exceeded, because, as discussed before, in a real reactor there will always be some particle to particle heat transfer leading to an ignition front moving toward the inlet of the reactor. Thus, as was already recognized by Eigenberger, but explicitly denied by Morbidelli et al., if multiplicity and ignition of the catalyst particles are to be expected, one should take particle to particle heat transfer into account, for example by including axial dispersion of heat.

\section{A. N. R. BOS \\ K. R. WESTERTERP ${ }^{\dagger}$}

\footnotetext{
Author to whom correspondence should be addressed.
}

Chemical Reaction Engineering Laboratories

Department of Chemical Engineering

Twente University, P.O. Box 217

7500 AE Enschede, The Netherlands

\section{REFERENCES}

Bauman, E. G. and Varma, A., 1990, Parametric sensitivity and runaway in eatalytic reactors. Chem. Engng $S c i .45$, 2133-2139.

Eigenberger, G., 1972, On the dynamic behavior of the catalytic fixed-bed reactor in the region of multiple steady states. I. Chem. Engny Sci. 27, 909-917.

Lee, C. K. and Varma, A., 1987, Steady state multiplicity behavior of an adiabatic plug-flow reactor with nonuniformly active catalyst. Chem. Engng Commun. 58, 287-309.

Lee, C. K. and Varma, A., 1988, An isothermal fixed-bed reactor with non-uniformly active catalyst. Chem. Engng Sci. 43, 1995-2000

Lee, C. K., Morbidelli, M. and Varma, A., 1987, Steady state multiplicity of an isothermal axial dispersion fixed-bed reactor with nonuniformly active catalyst. Chem. Engng Sci. 42, 1595-1608.

Liu, S. L. and Amundson, N. R., 1962, Stability of adiabatic packed bed reactor. An elementary treatment. Ind. Engng Chem. Fundam. 1, 200-208.

Morbidelli, M. and Varma, A., 1986a, Parametric sensitivity in fixed-bed catalytic reactors: the role of interparticle transfer resistances. A.I.Ch.E. J. 32, 297-306.

Morbidelli, M., Sarvido, A. and Varma, A., 1986b, Optimal catalyst activity profiles in pellets. 4 . Analytical evaluation of the isothermal fixed-bed reactor. Ind. Engng Chem. Fundam. 25, 307-313.
$0009-2509 / 91 \quad \$ 3.00+0.00$ Pergamon Press ple

\section{Authors' reply to comments by A. N. R. Bos and K. R. Westerterp}

(Received 13 December 1990; accepted 23 April 1991)

Dear Sirs,

The Implicit Function Theorem (IFT) provides sufficient conditions which guarantee that an implicit equation $f\left(0, \theta_{p}\right)$ $=0$, has a unique solution in a sufficiently small neighbourhood of a given solution $\left(\bar{\theta}, \bar{\theta}_{p}\right)$ i.e. the implicit equation can be made explicit as $\theta_{p}=\theta_{p}(\theta)$, along with some continuity characteristics for the function $\theta_{p}(\theta)$. The IFT does not say anything about the existence of other solutions away from the neighbourhood of $\left(\bar{\theta}, \bar{\theta}_{p}\right)$. This matter is discussed in more detail in Appendix B of Morbidelli et al. (1986b), to which the interested reader may refer for further details.

It should be obvious that since our reactor model describes physical quantities, we should choose, when they are available, continuous and continuously differentiable solutions, or as in the case under examination, solutions as less weak as possible. This brings us to the conclusion adopted in our work: the IFT by telling us under which conditions our model admits a continuous solution (or the less weak one), also forces us to choose under those conditions that solution. As a consequence, the problem of infinite solutions of the model is resolved, in the sense that we have a criterion for selecting the solution, except at the reactor inlet or at the bifurcation points. It should be quite obvious that, by doing this, we are not denying the existence of infinite solutions for the model equations! Note that during integration along the reactor axis, if the solution from the same branch (if availablc) were not followed, this would essentially imply that the reaction rate term, and consequently the first derivatives of the fluid phase concentration and temperature, would exhibit discontinuities along the reactor length. This is nonsensical for a physical problem.

We hope that this helps to further clarify what we did in our work, although it does not add anything beyond what we originally did, as all of this is reported in the Appendix noted above. We believe that the solution of the heterogeneous plug-flow model which we adopted in our series of papers is very useful, since resolving the infinite multiplicity problem makes it possible to work with such a modcl, which is very convenient since the model is simple and yet it captures most of the physical features of interest in applications. We did not deny that Eigenberger (1972) removed such a problem by accounting for heat conduction in the solid phase; we simply stated that this could also be done with a simpler model following our arguments.

There are some additional points in the letter of Bos and Westerterp $(B \& W)$, which need comment. 